\title{
Calculation of Magnetic Error Fields in Hybrid Insertion Devices
}

R. Savoy, K. Halbach, W. Hassenzahl, E. Hoyer, D. Humphries, and B. Kincaid,

\author{
Accelerator and Fusion Research Division \\ Lawrence Berkeley Laboratory \\ 1 Cyclotron Road \\ Berkeley, California 94720
}

August 1989 


\title{
Calculation of Magnetic Error Fields in Hybrid Insertion Devices
}

\author{
R. Savoy, K. Halbach, W. Hassenzahl, \\ E. Hoyer, D. Humphries, B. Kincaid \\ Lawrence Berkeley Laboratory, Berkeley, CA 94720
}

\begin{abstract}
The Advanced Light Source (ALS) at the Lawrence Berkeley Laboratory requires insertion devices with fields sufficiently accurate to take advantage of the small emittance of the ALS electron beam. To maintain the spectral performance of the synchrotron radiation and to limit steering effects on the electron beam these errors must be smaller than $0.25 \%$. This paper develops a procedure for calculating the steering error due to misalignment of the easy axis of the permanent magnet material. The procedure is based on a three dimensional theory of the design of hybrid insertion devices developed by one of us. The acceptable tolerance for easy axis misalignment is found for a $5 \mathrm{~cm}$ period undulator proposed for the ALS.
\end{abstract}

This work was supported by the Director, Office of Energy Research, Office of Basic Energy Sciences, Material Sciences Division, of the U.S. Department of Energy, under contract No. DE-AC03-76SF00098. 


\section{Introduction}

The demand for very high brightness synchrotron radiation will insure that insertion devices (IDs), wigglers and undulators, will be used extensively in the dedicated light sources that are under construction at present. The Advanced Light Source (ALS) project at the Lawrence Berkeley Laboratory is scheduled for completion in 1993. Based on input from the user community through a series of workshops and by letters of interest, we are designing a set of insertion devices and associated beam lines that span the spectral range accessible to the ALS. The first devices will have periods of $3.9,5.0,8.0$, and $13.6 \mathrm{~cm}$. The first three are undulators and will be about $4.5 \mathrm{~m}$ long; the $13.6 \mathrm{~cm}$ period ID will be a wiggler with a length of $2.5 \mathrm{~m}$. The first of these devices to be designed and constructed will be the $5 \mathrm{~cm}$ period undulator, U5.0. The examples used in the discussion of errors below will use the parameters of this device [1].

We have chosen to use a hybrid configuration with vanadium permendur poles and neodymium-iron-horon ( $\mathrm{Nd}-\mathrm{Fe}-\mathrm{B}$ ) permanent magnetic material to achieve the maximum possible performance in terms of peak field strength. Another advantage of the hybrid design is its reduced sensitivity to certain types of construction errors. There has been considerable advance in the understanding of these devices in the past five years. In this paper we use the theory $[2,3,4]$ developed by one of the authors to evaluate the effects of errors in the alignment of the easy axis of the charge sheet equivalent material (CSEM) on the magnetic fields in the insertion device and thus on the electron beam and on the synchrotron radiation that is produced. The reason for concern regarding the effects of this characteristic of the material on device performance 
is the observation of field errors localized in the region between poles in devices such as the Transverse Optical Klystron (TOK) at the NSLS at Brookhaven National Laboratory and the Beam Line $X$ Wiggler at the Stanford Synchrotron Radiation Laboratory [5].

\section{Discussion of error fields}

The magnetic field in an ideal undulator at the path of the electron beam has only a y component, and the electrons oscillate around a straight line in the $z$ direction. There is no net steering because the integral of the field component By alorig the electron beam path is zero. Real insertion devices have fabrication and assembly errors and are made with materials that have properties which vary slightly from sample to sample. These variations can accumulate in a statistical sense and will cause the beam to deviate from a straight average trajectory, leading to a reduced quality of the light produced [6]. The measure of the steering is given by the steering integral $\Delta \mathbf{S}$ :

$$
\Delta S=\int_{-\infty}^{+\infty} B_{y}(z) d z
$$

where $B_{y}$ is in Gauss (G) and $z$ in $\mathrm{cm}$. Each individual error: material, fabrication, or assembly, will contribute to the total steering integral.

Superposition hold's for the fields produced by small errors; thus, each error in each half period can be calculated separately and the results combined as needed for analysis of the devices. 
We consider here the error field component in the $y$ direction. The field component $B_{x}$ is assumed to be zero at the midplane because of symmetry, and because an error field component parallel to a steel surface is very small as long as the permeability in the steel is large enough. This imposes a boundary condition on the field that suppresses the $\mathrm{B}_{\mathrm{x}}$ component effectively. One of the major advantages of an hybrid insertion device compared with one made purely from permanent magnet material is this intrinsic suppression of $B_{x}$ and therefore the skew quadrupole.

The pole ${ }^{1}$ and the Nd-Fe-B blocks are shown in Fig. 1. The CSEM that energizes the pole consists of six square blocks, which overhang the pole on the sides and on the top. The overhangs were calculated using the three dimensional theory $[2,3,4]$ and are essential to achieve the design field. The blocks and the pole are held by an aluminum keeper, which is attached to a soft iron backing beam in a multi period aluminum assembly section $[7,8]$. A cross sectional side view of the device along the electron beam is shown in Fig. 2 .

The minimum gaps of the devices under consideration are small compared with the lateral $(x)$ width of the poles. The magnetic field within the devices is therefore approximately two dimensional and can be calculated by using two dimensional computer codes, for example POISSON or PANDIRA [9] in which it is sufficient to use a quarter period model because of symmetry. A consequence of the two dimensionality of the magnetic field is that the flux related to error fields is approximately given by the product of the steering

\footnotetext{
1 The vanadium permendur pole is sometimes referred to as as the steel pole.
} 
integral and the lateral pole width $w_{\text {pole: }}$

$$
\Delta \phi=\Delta S w_{\text {pole }}
$$

Error fields may deflect the electrons from their ideal path, resulting in a serious degradation of the synchrotron light output and may even affect the operation of the storage ring if the steering integral exceeds a certain value. The steering in the ALS insertion devices is specified for these reasons to be less than $500 \mathrm{Gcm}[8]$. These tight requirements can be achieved only if all possible error sources are carefully controlled.

The tool for analyzing the impact of construction, assembly, and material errors is a thisory for the design of hybrid insertion devices developed by one of the authors $[2,3,4]$. The theoretical considerations result in formulae that can be used for calculating the net error flux across the midplane of an insertion device. The following paragraphs outline the major ideas of this theory using as an example the error in alignment of the easy axis. The detailed discussion of other error sources, using this theor etical framework, can be found elsewhere [10].

\section{Error Fleids by Permanent Magnet Material Easy Axis Misalignment}

An ideal block of permanent magnet material is magnetized in the $z$ direction. Real material, however, shows a distribution of the magnetization direction around the ideal value. Typical materials show angular variations, $\alpha_{\text {, }}$ up to about \pm 3 deg. 
An error in the easy axis orientation of a block of CSEM can be modeled by charges on the top and the bottom surface of the block. The magnetic charge density $\sigma$ on these surfaces is given by:

$$
\sigma=B_{r} \sin \alpha
$$

where $B_{r}$ is the magnitude of the remanent field in the CSEM, $11.1 \mathrm{kG}$ for the Nd-Fe-B that we intend to use. The charge sheet on the top bounciary of the CSEM, far away from the electron beam and from the steel backing beam, produces a negligible contribution to the steering integral, whereas the charge sheet on the surface next to the beam may cause a considerable effect.

Steering due to the easy axis misalignment in a CSEM block having the same width as the steel pole

Magnetic charges cause two types of field: the direct field, which is associated with flux that originates on the charges when all poles and the midplane are on zero scalar potential, and the indirect field, which is due to flux associated with changes in scalar potential of the steel poles caused by the charges. The major part of the direct field is localized near the charge, as illustrated in Fig. 3a, whereas the indirect field is distributed over a much larger area (see Fig. 3b). The indirect flux is affected by the proximity of other steel structures such as the backing beam. 
Because there are no magnetic monopoles, the total flux, direct plus indirect, across any closed surface must be zero. Thus, the net flux across the midplane, that is, the sum of the direct and the indirect flux, is zero. However, there can be a difference between the integrals of the direct and the indirect fields along the electron path. This difference is the net steering integral.

The theory $[2,3,4]$ predicts that the direct flux across the midplane can be calculated by:

$$
\Delta \phi_{\text {direct }}=\int_{\text {Surface }} \sigma \frac{V\left(r_{q}\right)}{V_{0}} d A
$$

where $V\left(r_{q}\right)$ is the scalar potential at the location of the charge, $V_{0}$ is the potential of the midplane with all poles on zero scalar potential and $d A=w_{p o l e} d z$ is a surface element of the CSEM block. The integration is over those block surfaces that have a non zero surface charge. Assuming that the easy axis error is constant throughout the CSEM block, the steering integral due to charges on the CSEM surface closest to the electron beam is:

$$
\Delta S_{\text {direct }}=B_{r} \sin \alpha \int \frac{V\left(r_{q}\right)}{V_{0}} d z
$$

The integration on the right hand side of Eq. $4 a$ is along the bottom surface of the CSEM. 
The fraction of the indirect flux that is seen by the electron beam is determined by the geometry of the magnetic structure. This portion of the flux may be found by considering geometry dependent capacitances (analogous to electrical capacitances). The capacitance between the pole and the parts of the midplane directly under the pole is called $C_{F}$. The capacitance between the pole and all other surfaces is called $C_{5}$. The total capacitance is:

$$
C_{0}=C_{F}+C_{S}
$$

The total indirect flux is equal to the direct flux, therefore the indirect flux across the part of the midplane directly under the pole is:

$$
\Delta \phi_{\text {indirect }}=-\Delta \phi_{\text {direct }} \frac{C_{F}}{C_{0}}
$$

The capacitances are calculated using two dimensional computer codes. The result of these calculations is that the capacitance ratio $C_{F} / C_{0}$ for $U 5.0$ is 0.62 at the minimum gap. Details of the capacity calculations are described in [10].

The net steering integral is calculated from the net flux using Eqs. 2 and 6:

$$
\Delta \mathbf{S}_{\text {net }}=\left(1-\frac{C_{F}}{C_{0}}\right) \Delta S_{\text {direct }}
$$




\section{Effect of asymmetric errors}

The previous discussion is valid for a symmetrical easy axis error, that is, the error angles in the CSEM above the midplane and in the CSEM below the midplane are equal. Typically the error in the CSEM above the midplane will be different from that below. This asymmetric condition can be decomposed into symmetric and antisymmetric parts, as shown in Fig. 4 (Note: The symmotry characteristic of an ideal insertion device is such that a positive charge above the midplane has an equivalent negative charge below the midplane. This is the same condition as a symmetrical error). Only the symmetric charge distribution produces field components that can steer the beam. An asymmetric easy axis error with angle $\alpha$ produces the same steering as a symmetric configuration with error angle $\alpha / 2$.

The integral of the scalar potential ratio in Eq. $4 a$ can be calculated with a POISSON run, with all poles on zero scalar potential and the midplane on scalar potential $V_{0}$. A typical field plot of this scalar potential run is shown in Fig. 5, where the upper half of a quarter period of the device is modeled. The boundary conditions are set so that the scalar potential lines are perpendicular to the side boundaries and parallel to the top and bottom boundary. The scalar potential along the bottom surface of the CSEM block, integrated over a quarter period, is multiplied by two to evaluate the effect for a half period. The steering integral due to the direct field is calculated with Eq. 4a:

$$
\Delta S_{\text {direct }}=4329 \frac{\mathrm{Gcm}}{\mathrm{rad}} \Delta \alpha
$$


where $\alpha$ is in radians (for small angles). The net steering integral for a symmetric configuration is

$$
\Delta S_{\text {net }}=1645 \frac{\mathrm{Gcm}}{\mathrm{rad}} \Delta \alpha
$$

This result corresponds to equal lateral widths of the steel poles and the CSEM blocks.

Steering due to the easy axis misalignment in segmented CSEM blocks including the effect of block overhang at the side

The steering integral due to an easy axis error in the cencer block is different from that in a side block because the direct field of a side block does not influence the electron beam. The direct ar $t$ indirect flux due to a misalignment in the center block is:

$$
-\Delta \phi_{\text {indirect }}=\Delta \phi_{\text {direct }}=\Delta S_{\text {direct }} W_{\text {block }}
$$

The indirect flux under the pole is distributed across the entire pole width. Thus:

$$
\Delta S_{\text {indirect }}=\frac{\Delta \phi_{\text {indirect }}}{W_{\text {pole }}} \frac{C_{F}}{C_{0}}=-\Delta S_{\text {direct }} \frac{W_{\text {block }}}{W_{\text {pole }}} \frac{C_{F}}{C_{0}}
$$


The net steering is given by the sum of the direct and indirect contribution:

$$
\Delta S_{\text {net }}=\Delta S_{\text {direct }}\left(1-\frac{W_{\text {block }}}{W_{\text {pole }}} \frac{C_{F}}{C_{0}}\right)
$$

With $w_{\text {pole }}=8 \mathrm{~cm}$ and $w_{b l o c k}=3.5 \mathrm{~cm}$ the net flux at the beam due to a symmetrical easy axis error in the center blocks is $+3155 \mathrm{Gcm} / \mathrm{rad}$.

Easy axis orientation errors in the side blocks only produce indirect field errors at the electron beam position. However, the direct flux (and thus the indirect flux also) of the side block is larger than that of the center block because of the overhang. We take this into account by estimating that the effective block width is about $50 \%$ larger than wblock, resulting in

$$
\Delta \mathbf{S}_{\text {net }}=-\Delta \mathbf{S}_{\text {direct }} \frac{C_{F}}{C_{0}} \frac{1.5 W_{\text {block }}}{W_{\text {pole }}}=-1761 \frac{\mathrm{Gcm}}{\mathrm{rad}}
$$

This error, $-1761 \mathrm{Gcm} / \mathrm{rad}$, is about the same as in the case when there is only one CSEM block over the whole width of the iron pole. 


\section{Results and Discussion}

The importance of any construction error is found by comparing the resulting error flux to the total ideal flux in a half period, $13060 \mathrm{Gcm}$. An acceptable error in each half period of the U5.0 undulator due to all causes is $0.25 \%(33 \mathrm{Gcm})$. Since the $0.25 \%$ must be budgeted among the various independent errors, it is convenient to determine the magnitude of each error that will cause a $0.1 \%$ error in the flux. This corresponds to an asymmetrical misalignment error of either $0.5 \mathrm{deg}$ in the center block or $0.9 \mathrm{deg}$ in a side block. It is somewhat surprising that the effect of equal misorientation in all blocks leads to a very small steering error of $-367 \mathrm{Gcm} / \mathrm{rad}$, whereas the two dimensional case gave $+1645 \mathrm{Gcm} / \mathrm{rad}$. The difference is due entirely to the estimated effect of the block overhang.

The resulting requirement on CSEM material is much tighter than can be met with the usual fabrication processes. From measurements reported elsewhere [11] relating surface easy axis orientation and bulk moments we know that the easy axis orientation is not uniform in a single block. Therefore, the easy axis orientation at the surface (i.e. the magnetic surface charge distribution) must be measured and the blocks sorted accordingly. 


\section{References}

[1] W.Hassenzahi, J.Chin, K.Halbach, E.Hoyer, D.Humphries, B.Kincaid, R.Savoy, Insertion Devices for the Advanced Light Source at LBL, US Part. Acc. Conf. Chicago 1989 (LBL Report 25992, 1989) to be published in IEEE Trans. Nucl. Sci., Vol. NS-36, 1989

[2] K.Halbach, Design of Magnets, Lecture Series at KfA Jülich, May/June 1985

[3] K.Halbach, Design of Hybrid Insertion Devices, Lecture Series given at LBL, Oct 1988 March 1989, ensemble of reports and video tapes, LBL V-8811

[4] K.Halbach, LBL, ALS-Note LSBL-034, 7/1/1989

[5] E.Hoyer, K.Halbach, D.Humphries, S.Marks, D.Plate, D.Shuman, V.P.Karpenko, S.Kulkarni, K.G.Tirsell, The Beam Line X NdFe-Steel Wiggler for SSRL, IEEE Catalogue No.87, Chap. 2387-9, 1987, p 1508

[6] B.Kincaid, Random errors in undulators and their effect on the radiation spectrum, J. Opt.Soc.Am. B, Vol. 2, 1294-1306, 1985

[7] E.Hoyer, J.Chin, K.Halbach, W.Hassenzahl, D.Humphries, B.Kincaid, H.Lancaster, D.Plate, R.Savoy, The U5.0 Undulaiser Design for the Advanced Light Source at LBL, companion paper this conference

[8] E.Hoyer, J.Chin, K.Halbach, W.Hassenzahl, D.Humphries, B.Kincaid, H.Lancaster, D.Plate, R.Savoy, U5.0 Undulator Conceptual Design Report, to be published, 1989

[9] PANDIRA is a modified version of POISSON, it allows the solution of permanent magnet and residual field problems. POISSON is a improved version of TRIM (A.M.Winislow, J.Computer Phys. $1,149,1967)$ that was developed by K.Halbach et al.

[10] K.Halbach, W.Hassenzahl, R.Savoy, Error calculations in hybrid insertion devices, to be published

[11] W.Hassenzahl, D.Nelson, Characterizing Blocks of Permanent Magnet Materials, companion paper this conterence 
Figure captlons

Figure 1: View of one vanadium permendur pole with CSEM blocks

Figure 2: Side view of a part of the device

Figure 3: Direct and indirect fields for a typical insertion device

Figure 4: Decomposition of an asymmetric charge distribution (a) into antisymmetric (b) and symmetric (c) charge distributions

Figure 5: Scalar potential field plot used to calculate the effect of construction errors on the steering integral 


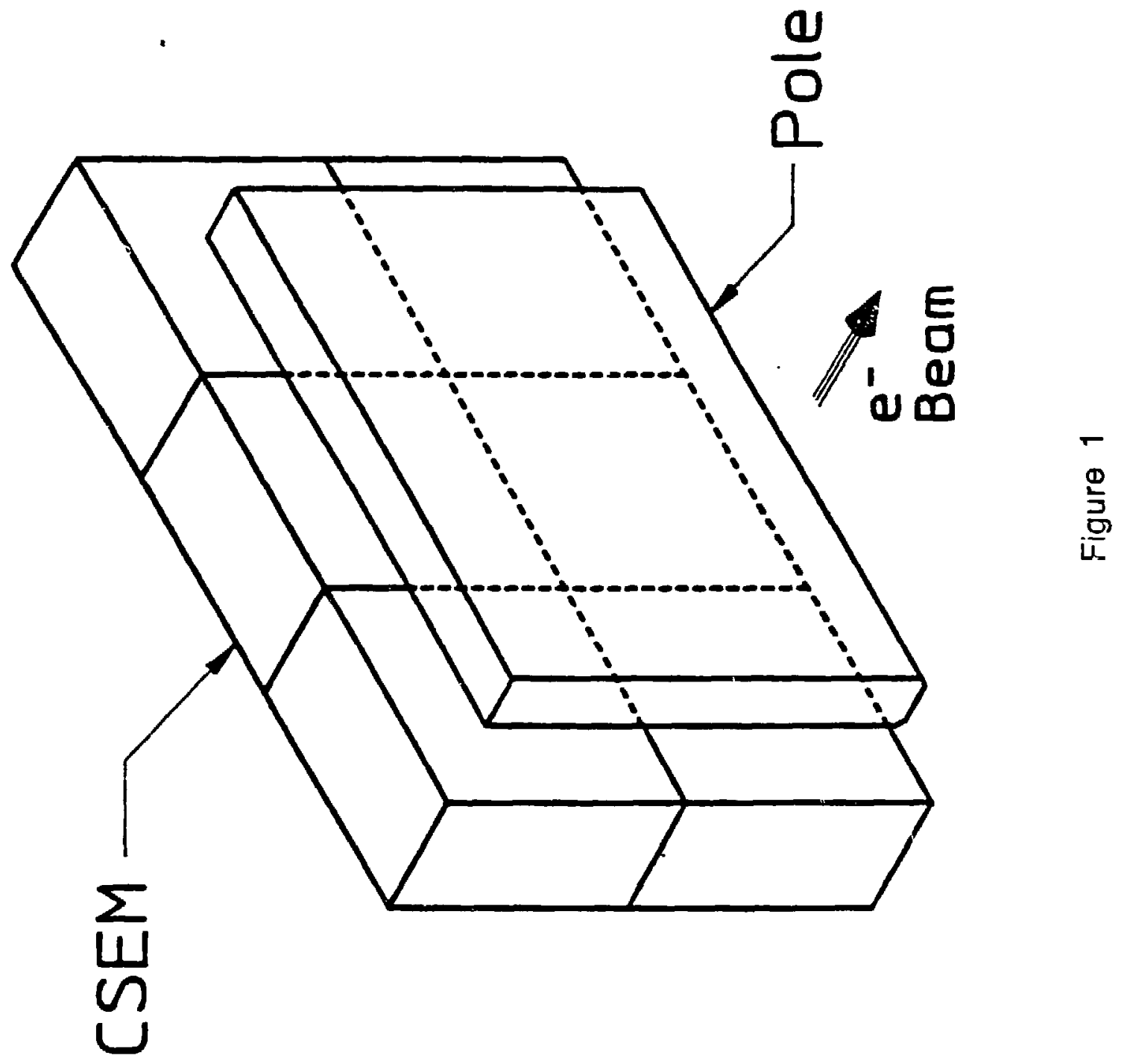




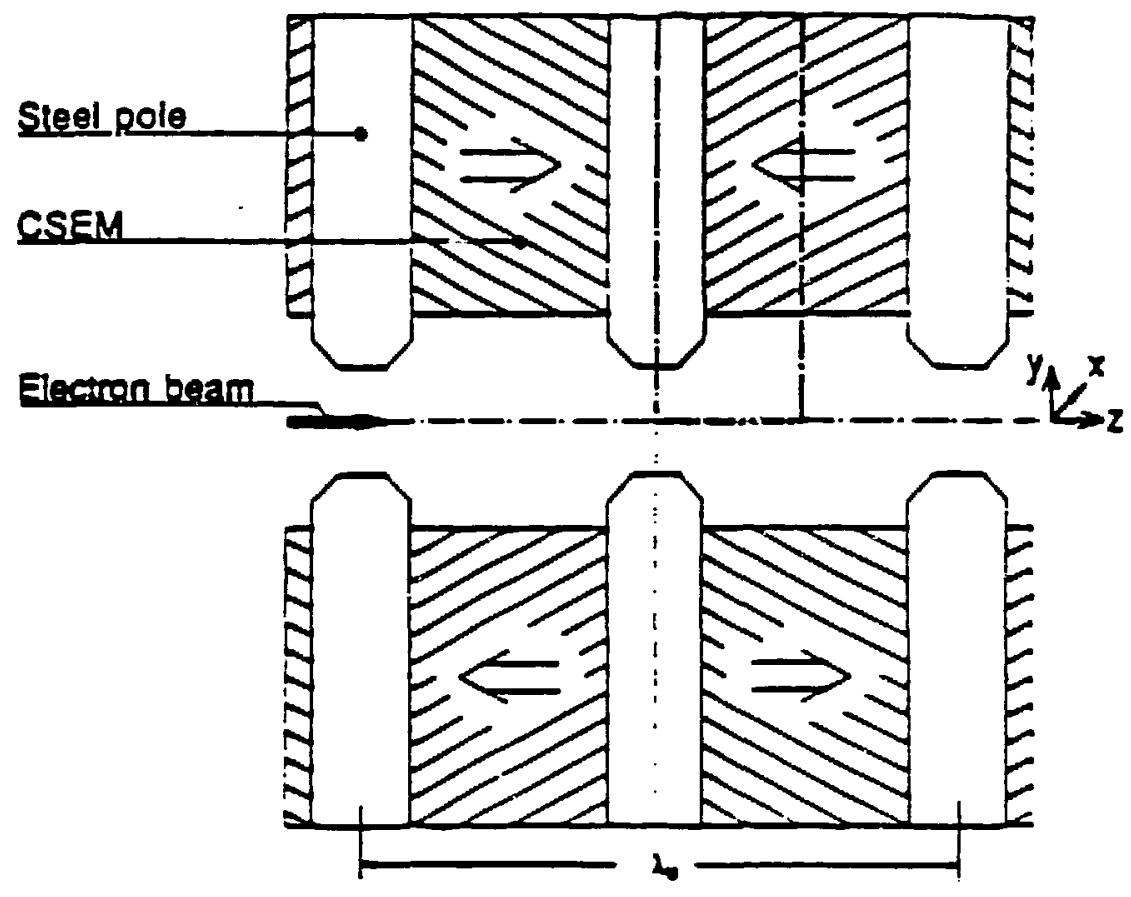

Figure 2 


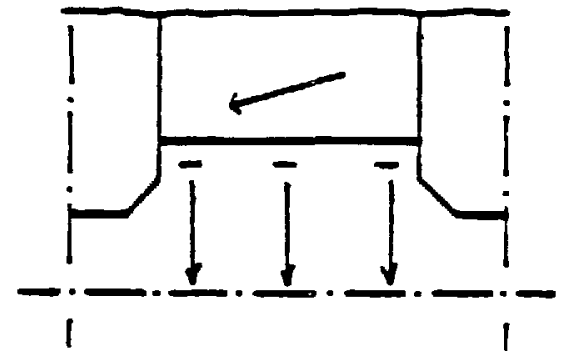

Side view

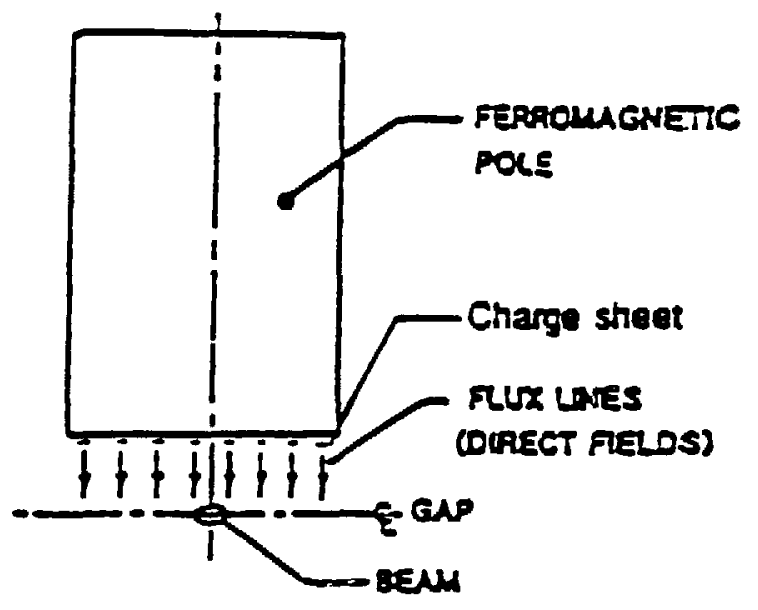

a

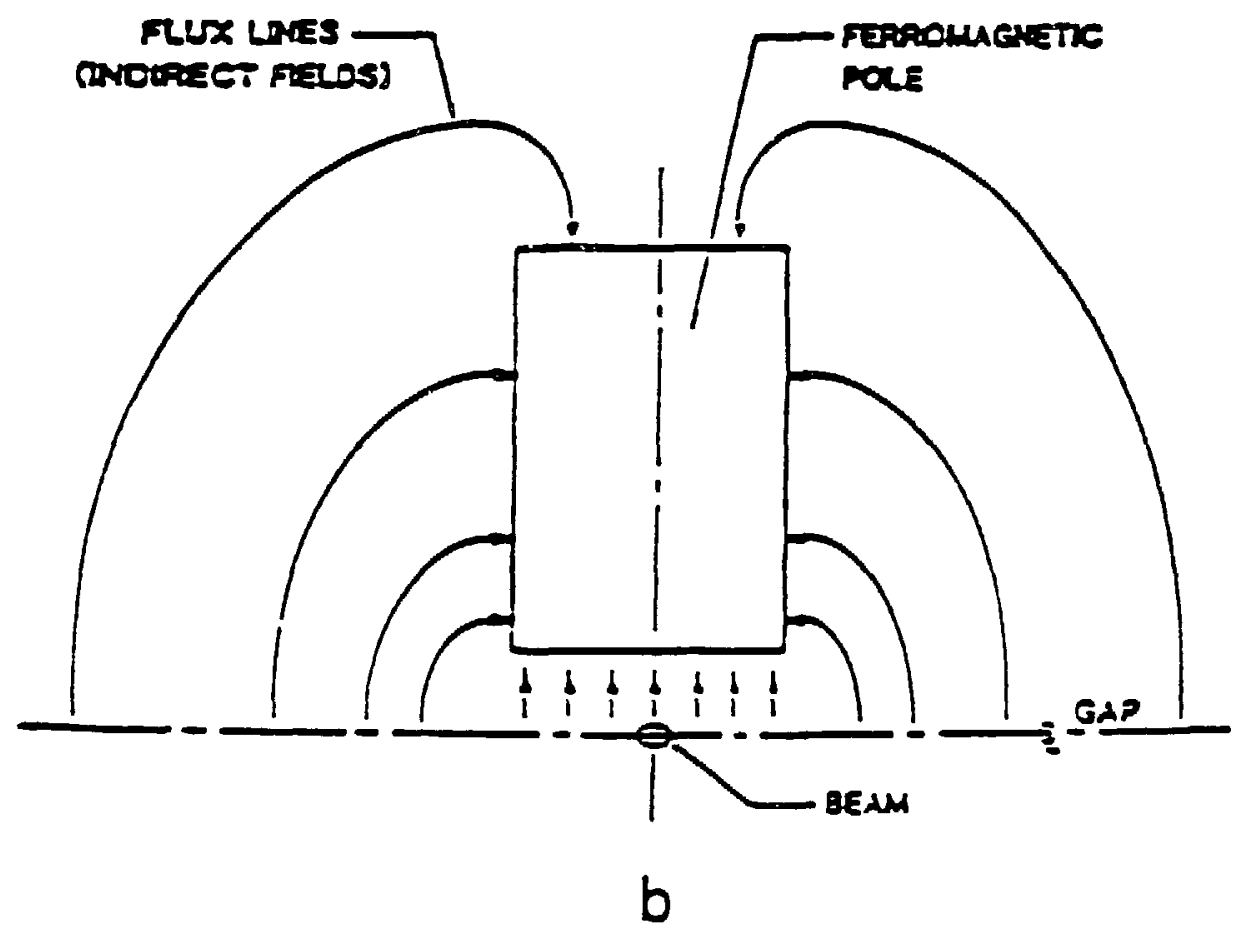

Figure 3 


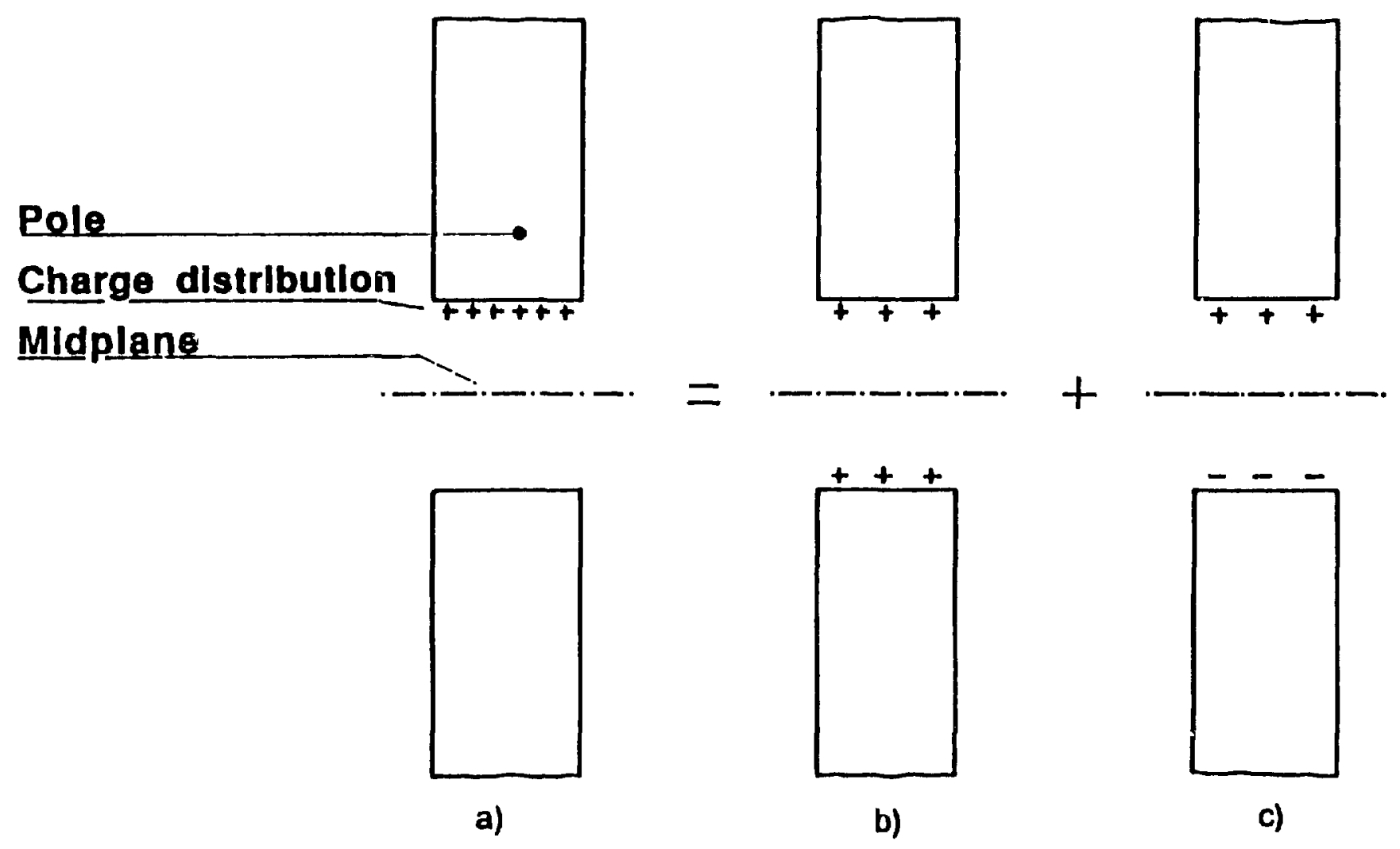

Figure 4 


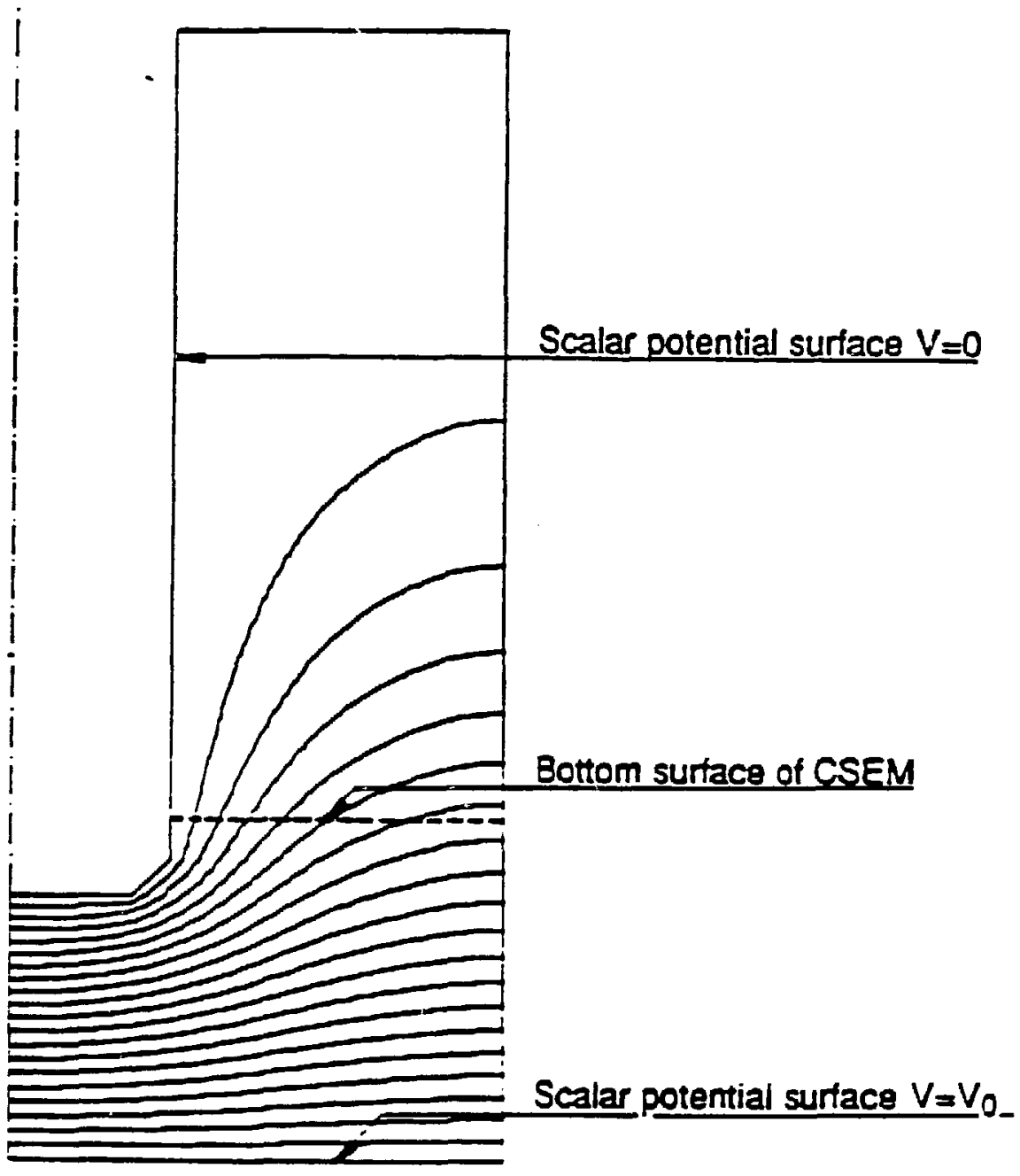

Figure 5 


\section{DISCLAINIER}

This document was prepared as an account of work sponsored bi the Linted Stules Government. Vetther the Linited States Guvernment nor anv agency thereot. nor The Regents of the Lnversty of Calitumia. nor any of their employees, makes any warranty express or implied. or assumes any legal liability or responsibility for the accuracy, completeness, or usefulness of any information. apparatus. product. or process disclosed. or represents that its use would not infringe privately owned rights. Reference herein to any specific commercial products process. or service by its trade name, trademark. manufacturer. or otherw'se: does not necessanly constitute or imply its endorsement. recommendation. or favoring by the United States Government or an! agenc! thereof. or The Regents of the Universily of Californa. The view's and epinions of authors expressed herein do not necessanly state or reflect those of the linited States Goternment or any agency thereof or The Regents of the 1 nuersity of Calıforna and shall not be used for adverising or product indorsement purposes.

Lawrence Berkeley Laboratory is an equal opportunity employer 